Brazilian Journal of Medical and Biological Research (2009) 42: 470

ISSN 0100-879X

\title{
Correction
}

Braz J Med Biol Res 2004; 37(10): 1551-1556

\section{Effect of the aerobic capacity on the validity of the anaerobic threshold for determination of the maximal lactate steady state in cycling}

B.S. Denadai, T.R. Figueira, O.R.P. Favaro and M. Gonçalves

Page 1551. The correct list of authors is printed above.

The correct surname of second author is Figueira.

For html or pdf file, see: http://www.scielo.br/bjmbr 